YITP-SB-05-50

\title{
Fragmentation, NRQCD and Factorization in Heavy Quarkonium Production
}

\author{
Gouranga C. Nayak*, Jian-Wei Qiu ${ }^{\dagger}$ and George Sterman* \\ ${ }^{*}$ C.N. Yang Institute for Theoretical Physics, Stony Brook University, SUNY, Stony Brook, New \\ York 11794-3840, U.S.A. \\ ${ }^{\dagger}$ Department of Physics and Astronomy, Iowa State University, Ames, Iowa 50011-3160, U.S.A.
}

\begin{abstract}
We discuss factorization in heavy quarkonium production in high energy collisions using NRQCD. Infrared divergences at NNLO are not matched by conventional NRQCD matrix elements. However, we show that gauge invariance and factorization require that conventional NRQCD production matrix elements be modified to include Wilson lines or non-abelian gauge links. With this modification NRQCD factorization for heavy quarkonium production is restored at NNLO.
\end{abstract}

\section{INTRODUCTION}

Quarkonium production and decay have been the subject of a vast theoretical literature and of intensive experimental study [1], in which the effective field theory nonrelativistic QCD (NRQCD) [2] has played a guiding role. NRQCD offers a systematic formalism to separate dynamics at the perturbative mass scale of the heavy quarks from nonperturbative dynamics, through an expansion in relative velocity within the pair forming the bound state. An early success of NRQCD was to provide a framework for the Tevatron Run I data on high- $p_{T}$ heavy quarkonium production [3], and it has been extensively applied to heavy quarkonia in both collider and fixed target experiments [1].

In contrast to quarkonium decay, fully convincing arguments have not yet been given for NRQCD factorization as it is applied to high- $p_{T}$ production processes. Here we review new tests of NRQCD factorization for high- $p_{T}$ quarkonia production at next-tonext-to-leading order (NNLO) [4]. At NNLO we find infrared divergences that do not fall precisely into the pattern suggested in Ref. [2]. These divergences may, however, be incorporated into color octet matrix elements by a technical redefinition in which Wilson lines which makes them gauge invariant, restore factorization at NNLO.

If we assume NRQCD factorization, we have

$$
d \sigma_{A+B \rightarrow H+X}\left(p_{T}\right)=\sum_{n} d \hat{\sigma}_{A+B \rightarrow c \bar{c}[n]+X}\left(p_{T}\right)\left\langle\mathscr{O}_{n}^{H}\right\rangle,
$$

where the $\mathscr{O}_{n}^{H}$ are NRQCD operators for state $H$, which were introduced in Ref. [2] in the form

$$
\mathscr{O}_{n}^{H}(0)=\chi^{\dagger} \mathscr{K}_{n} \psi(0)\left(a_{H}^{\dagger} a_{H}\right) \psi^{\dagger} \mathscr{K}_{n}^{\prime} \chi(0),
$$




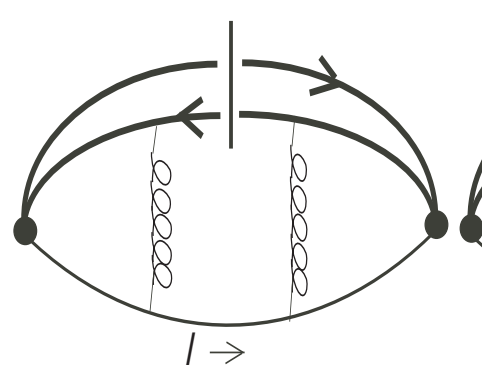

(a)

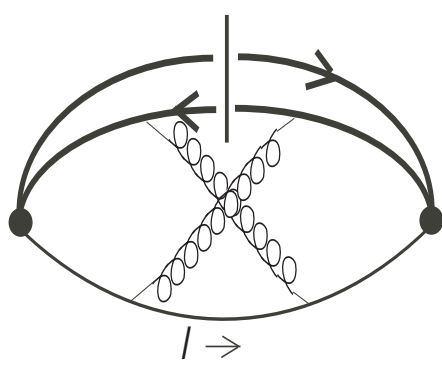

(b)

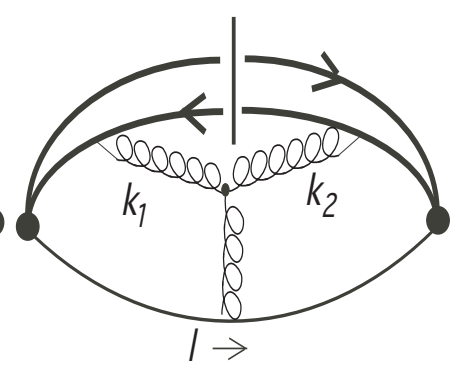

(c)

FIGURE 1. Representative NNLO contributions to $g \rightarrow c \bar{c}$ fragmentation in eikonal approximation.

where $a_{H}^{\dagger}$ is the creation operator for state $H, \chi(\psi)$ are two component Dirac spinors and where $\mathscr{K}_{n}$ and $\mathscr{K}_{n}^{\prime}$ involve products of color and spin matrices, and at higher dimensions of covariant derivatives.

Our gauge invariant redefinition of production operators in octet representation is the replacement (which we refer to as a gauge completion),

$$
\mathscr{O}_{n}^{H}(0) \rightarrow \chi^{\dagger} \mathscr{K}_{n, c} \psi(0) \Phi_{l}^{\dagger}[0, A]_{c b}\left(a_{H}^{\dagger} a_{H}\right) \Phi_{l}[0, A]_{b a} \chi^{\dagger} \mathscr{K}_{n, a}^{\prime} \psi(0),
$$

where we have exhibited the color indices of the octets. Here

$$
\Phi_{l}[0, A]=\mathscr{P} \exp \left[-i g \int_{0}^{\infty} d \lambda l \cdot A(\lambda l)\right]
$$

is a Wilson line or gauge link, with $l^{2}=0$. The field $A=A^{a} T^{a}$ with $T^{a}$ being the generator in the adjoint representation.

Representative diagrams for the fragmentation function at NNLO are shown in Fig. 1 in which the off shell lines are indicated by the heavy dots. The full infrared fragmentation function is generated by taking all allowed cuts of the remaining lines of each such diagram. We are concerned only with diagrams that connect octet to singlet quark states, and which are not topologically factorized, since these are the potential sources of nonfactoring behavior in both the fragmentation function and related cross sections. The original argument for NRQCD factorization was based on the conjecture that all infrared regions in these diagrams cancel after this limited sum over cuts [2]. In fact, this is the case at NNLO only if we employ the gauge-completed definitions for NRQCD matrix elements, as in Eq. (3) above.

The individual classes of diagrams in Fig. 1a and 1b, for which two gluons are exchanged between the quarks and the Wilson line, satisfy the infrared cancellation conjecture of Ref. [2], by summing over the possible cuts and connections to quark and antiquark lines, as do diagrams that have three gluon-eikonal vertices on the quark pair and one on the Wilson line. For the class of diagrams related to Fig. 1c, however, with a three-gluon interaction, this cancellation fails. Expanding to second order in the relative momentum $q$, the full contribution from Fig. 1c, found by cutting the gluon line $k_{1}$ and the Wilson line, can be written as 


$$
\begin{aligned}
\Sigma^{(2)}(q, l)=-16 i g^{4} \mu^{4 \varepsilon} & \int \frac{d^{D} k_{1}}{(2 \pi)^{D}} \frac{d^{D} k_{2}}{(2 \pi)^{D}} 2 \pi \delta\left(k_{1}^{2}\right) l^{\lambda} V_{v \mu \lambda}\left[k_{1}, k_{2}\right] \\
& \times\left[q^{\mu}\left(P \cdot k_{1}\right)-\left(q \cdot k_{1}\right) P^{\mu}\right]\left[q^{v}\left(P \cdot k_{1}\right)-\left(q \cdot k_{2}\right) P^{v}\right] \\
& \times \frac{1}{\left[P \cdot k_{1}+i \varepsilon\right]^{2}\left[P \cdot k_{2}-i \varepsilon\right]^{2}} \\
& \times \frac{1}{\left[k_{2}^{2}-i \varepsilon\right]\left[\left(k_{2}-k_{1}\right)^{2}-i \varepsilon\right]\left[l \cdot\left(k_{1}-k_{2}\right)-i \varepsilon\right]},
\end{aligned}
$$

where $V_{v \mu \lambda}\left[k_{1}, k_{2}\right]$ represents the momentum part of the three-gluon vertex. Summing over all contributions, however, we find a noncancelling real infrared pole in the fragmentation function (in the quarkonium rest frame $\vec{P}=0$ ) [4],

$$
\Sigma(P, q, l)=\alpha_{s}^{2} \frac{4}{3 \varepsilon} \frac{\vec{q}^{2}}{4 m_{c}^{2}}=\alpha_{s}^{2} \frac{1}{3 \varepsilon} \frac{\vec{v}^{2}}{4},
$$

where $\vec{v}$ is the relative velocity of the heavy quark pair.

Eq. (6) shows explicitly the breakdown of topological factorization of infrared dependence at NNLO. Its presence implies that infrared poles would appear in coefficient functions at NNLO and beyond when the factorization is carried out with octet NRQCD matrix elements defined in the conventional manner, Eq. (2). On the other hand, when defined according to its gauge-completed form (3), each octet NRQCD matrix element itself generates precisely the same pole terms given in (6) above. Thus, at least at NNLO and to order $v^{2}$, when the non-perturbative NRQCD matrix are defined according to Eq. (3), the factorization is restored. We are investigating the extensions of this result.

\section{ACKNOWLEDGMENTS}

This work was supported in part by the National Science Foundation, grants PHY0071027, PHY-0098527 and PHY-0354776, and by the Department of Energy, grant DE-FG02-87ER40371.

\section{REFERENCES}

1. N. Brambilla et al. (Quarkonium Working Group) hep-ph/0412158

2. G.T. Bodwin, E. Braaten and G.P. Lepage, Phys. Rev. D51, 1125 (1995), Erratum ibid. D55, 5853 (1997) hep-ph/9407339

3. CDF Collaboration (F. Abe et al.) Phys. Rev. Lett. 79, 572 (1997); CDF Collaboration (F. Abe et al.), ibid. 578.

4. G. C. Nayak, J. Qiu and G. Sterman, Phys. Lett. B613 (2005) 45; Phys. Rev. D 72 (2005) 114012. 